\title{
A two-directional 1-gram visual motion sensor inspired by the fly's eye
}

\author{
Frédéric L. Roubieu, Student Member, IEEE, Fabien Expert, Student Member, IEEE, Guillaume Sabiron, Student \\ Member, IEEE, and Franck Ruffier, Member, IEEE
}

\begin{abstract}
Optic flow based autopilots for Micro-Aerial Vehicles (MAVs) need lightweight, low-power sensors to be able to fly safely through unknown environments. The new tiny 6pixel visual motion sensor presented here meets these demanding requirements in term of its mass, size and power consumption. This 1-gram, low-power, fly-inspired sensor accurately gauges the visual motion using only this 6-pixel array with two different panoramas and illuminance conditions. The new visual motion sensor's output results from a smart combination of the information collected by several 2-pixel Local Motion Sensors (LMSs), based on the "time of travel" scheme originally inspired by the common housefly's Elementary Motion Detector (EMD) neurons. The proposed sensory fusion method enables the new visual sensor to measure the visual angular speed and determine the main direction of the visual motion without any prior knowledge. By computing the median value of the output from several LMSs, we also ended up with a more robust, more accurate and more frequently refreshed measurement of the 1-D angular speed.
\end{abstract}

Index Terms-Optic flow, Vision, Fly, Bio-inspiration, Neuromorphic, Motion sensor.

\section{INTRODUCTION}

$\mathbf{M}$ ICRO AERIAL VEHICLES (MAVs) constitute a class of Unmanned Aerial Vehicles (UAVs) which can be used for the remote observation of hazardous environments without any risk to human life. MAVs need to be equipped with onboard sensors and flight control devices in order to perform tasks such as those performed by optic flow (OF) based aerial robots: obstacle avoidance [1]-[7], terrain following and automatic landing [2], [8]-[12], tracking a moving target [13], [14] and controlling their forward speed [15]. MAVs endowed with these abilities would acquire greater autonomy, and at the same time, the ground operators' arduous task of piloting an almost constantly invisible aircraft would be greatly simplified. Nature has taught us that flying insects, which came into

Copyright(c) 2012 IEEE. Personal use of this material is permitted. However, permission to use this material for any other purposes must be obtained from the IEEE by sending a request to pubs-permissions@ieee.org.

An earlier version of this paper was presented at the 2011 IEEE SENSORS Conference and was published in its proceedings.

This research was supported partly by CNRS Institutes (Life Science; Information Science; Engineering Science and Technology), Aix-Marseille University, the French National Research Agency (ANR) (EVA project under ANR-ContInt grant number ANR608-CORD-007-04), and by European Commission via the CURVACE project. The CURVACE project acknowledges the financial support of the European Commission's Future and Emerging Programme for Research, under FET-Open grant number: 237940.

The authors are with Aix-Marseille University, CNRS, Institute of Movement Science, Biorobotics Dept. UMR7287, 13288, Marseille, France e-mail: \{frederic.roubieu, fabien.expert, guillaume.sabiron, franck.ruffier\}@univamu.fr.

G. Sabiron is also with the French Aerospace Lab (ONERA, Systems Control and Flight Dynamics -DCSD-), 31055 Toulouse, France. existence several hundred million years ago, have developed particularly elegant solutions to the problem of navigating swiftly in unfamiliar and complex environments. Winged insects are able to enter and explore unknown environments without any sonar or laser range-finder: their visually guided performances depend mainly on OF sensing processes [10], [16]-[25]. The OF perceived by a moving agent (an animal, human or robot) is a vector field that gives the angular speed $\omega$ (magnitude in $\% / s$ ) at which any contrasting object in the environment is moving past the eyes [26]. The fly's eye has been shown to be sensitive to two-directional motion [27] and also to be driven by a minimum of two photoreceptors inside the same ommatidium [28], [29]. The fly's eye is therefore one of the most suitable animal model available for studies on motion detecting neurons. Based on studies on the fly's visual system previously conducted at our Laboratory, in which electrophysiological recordings were performed on single neurons while microstimuli were being applied to single photoreceptor cells in a single ommatidium of the compound eye [28], a 2-pixel Local Motion Sensor (LMS) was developed [30], based on the principle known today as the "time of travel" scheme [31].

Using such bioinspired sensors, various simulated vision-based autopilots [31]-[34] based on OF sensing techniques were subsequently developed at our Laboratory, and a series of terrestrial [31], [35] and aerial robots [9], [14], [36], [37] were constructed. The "robotfly" ("Robot Mouche" in French) built by Franceschini's team in 1991 was a completely autonomous wheeled robot equipped with a compound eye consisting of 114 electronic LMSs implemented in analog technology using Surface Mounted Devices (SMDs) [35]. The "robotfly" was able to steer its way through an unknown field full of obstacles at a relatively high speed (up to $50 \mathrm{~cm} / \mathrm{s}$ ) [35]. The "robotfly" also implemented two-directional analog LMSs using a maximum operator to determine the direction of motion [35], [38]. However, the size and mass of these analog sensors were not compatible with the drastic constraints imposed on free flying MAVs in terms of their mass (they have to weigh less than $100 \mathrm{~g}$ ), size (they must measure less than $15 \mathrm{~cm}$ ) and power consumption.

Several teams therefore started to design new visual motion sensors by mixing analog and digital processing, which are lighter and easier to implement onboard MAVs than a camerabased system [39] or fully analog sensors. One possible approach consisted in developing visual motion sensors using analog and digital Very-Large-Scale Integration (VLSI) technologies, such as those based on the Reichardt correlator [40]- 


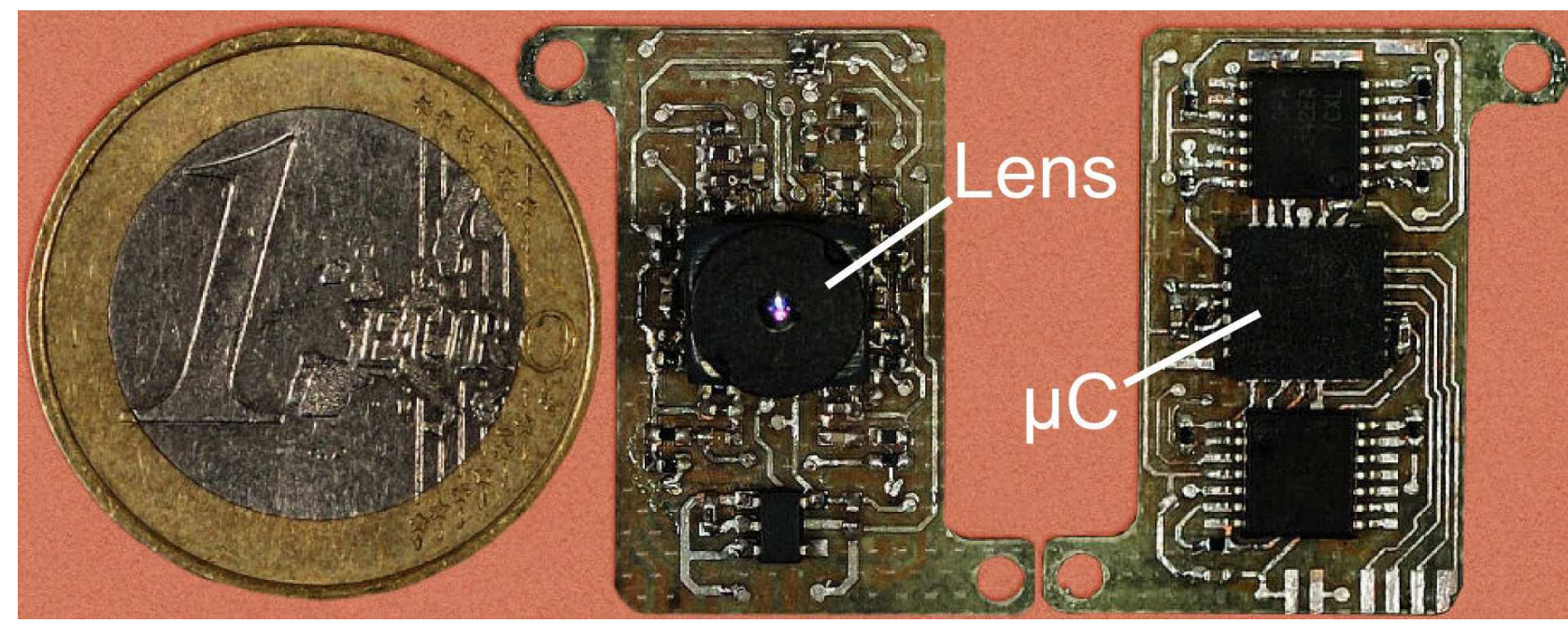

Fig. 1. Top view of the 1-gram microcontroller-based visual motion sensor (size: $23.3 \times 12.3 \mathrm{~mm}$ ) with its lens (focal length: $2 \mathrm{~mm}$ ) mounted on the one-dimensional 6-photosensor array, and bottom view of the PCB (thickness: $0.4 \mathrm{~mm}$ ) with its tiny low-power 16-bit $\mu C$ (dsPIC from Microchip $($ Company).

[43], the Pulse-based velocity sensor [44] or Barrows' design [45]. Only a few VLSI-based sensors have been implemented onboard MAVs so far (flight with limited degrees of freedom: [46], free-flight: [2], [45]).

Off-the-shelf mouse sensors were also recently characterized [47] and mounted onboard terrestrial [48], [49] and aerial robotic platforms [5], [50]. The performances of these systems have not been properly assessed so far in terms of their resolution, accuracy, invariance to illuminance and contrast, apart from two studies [51], [52].

At our Laboratory, several versions of 2-pixel motion sensors based on the "time of travel" scheme originally based on the fly's eye [29] were developed using either a Field Programmable Gate Array (FPGA) [53] or a microcontroller $(\mu C)$ [54]-[60].

In the current study, we present a new tiny $\mu C$-based visual motion sensor weighing only 1 gram (Fig. 1), which receives visual inputs from a 6-pixel array integrated circuit. By combining several 2-pixel motion sensors, the performances of the visual motion sensor were highly improved. The first sensory fusion method of this sensor produces a combined output based on the median value of 5 LMS measurements in a single pre-determined direction of motion which drastically improved the accuracy and the refresh rate $\left(f_{\text {refresh }}\right)$ of the angular speed measurements [58]. An improved sensory fusion method determines an accurate estimation of the direction and the magnitude of the angular speed in the detected direction of motion. This whole processing was embedded into a $\mu C$ which has sufficient computational resources for carrying out the requisite signal processing tasks efficiently, while its mass is compatible with the very low avionic payload allowed on MAVs.

The first sensory fusion method implemented in our tiny $\mu C$ based visual motion sensor is presented in the section II that gives a short description of the bio-inspired visual system and the principles underlying the 2-pixel "time of travel" scheme. Experiments performed on the visual motion sensor, which was tested indoors, are described in Section III. The results of these experiments are presented in Section IV. Section V describes the results obtained thanks to an improved sensory fusion method able to perfectly determine the direction of motion without any prior knowledge and to give an accurate and robust assessment of the magnitude of the motion in term of angular speed.

\section{DesCription OF THE DEVICE}

1) Photoreceptor configuration: The front end of the visual motion sensor designed and developed in this study was based on an off-the-shelf photosensor array (iC-LSC from iCHaus Company, http://www.ichaus.de) consisting of 2 rows of 6 photodiodes. A fixed-gain current amplifier is integrated into each photodiode. In order to detect a large number of contrasting objects at low illuminance levels, the photosensors in each column were paired to increase the signal to noise ratio by increasing the sensitive surface two-fold from $300 \mu m \times 800 \mu m$ to $300 \mu \mathrm{m} \times 1600 \mu \mathrm{m}$. This one-dimensional 6-pixel array was then mounted on a cheap, lightweight lens (Sparkfun SEN00637) borrowed from a mobile telephone camera (Fig. 1).

As in flies, each photosensor features a Gaussian Angular Sensitivity Function (ASF), [61] [Fig. 2(b)], which results in insects from the spatial convolution of the photoreceptor's diameter with the point spread function of the facet lenslet [62], [63] and in our sensor, from the defocusing of the lenslet. The ASF of the "lens-photoreceptor" system was assessed by slowly rotating the visual motion sensor placed $50 \mathrm{~cm}$ in front of a point light source [Fig. 2(a)]. By defocusing the lens (i.e., by reducing the distance between the lens and the retina), we obtained a similar Gaussian sensitivity profile to that of the housefly. The full width at half height of the Gaussian curve (the acceptance angle) $\Delta \rho$ determines the cutoff frequency of the low-pass spatial filtering process (Fig. 3), whereas the inter-receptor angle $\Delta \varphi$ (i.e., the angle between two adjacent optical axes) determines the angular speed $\left(\omega_{i}^{m}\right)$ measurement range.

The defocusing process was adjusted to obtain an appropriate 

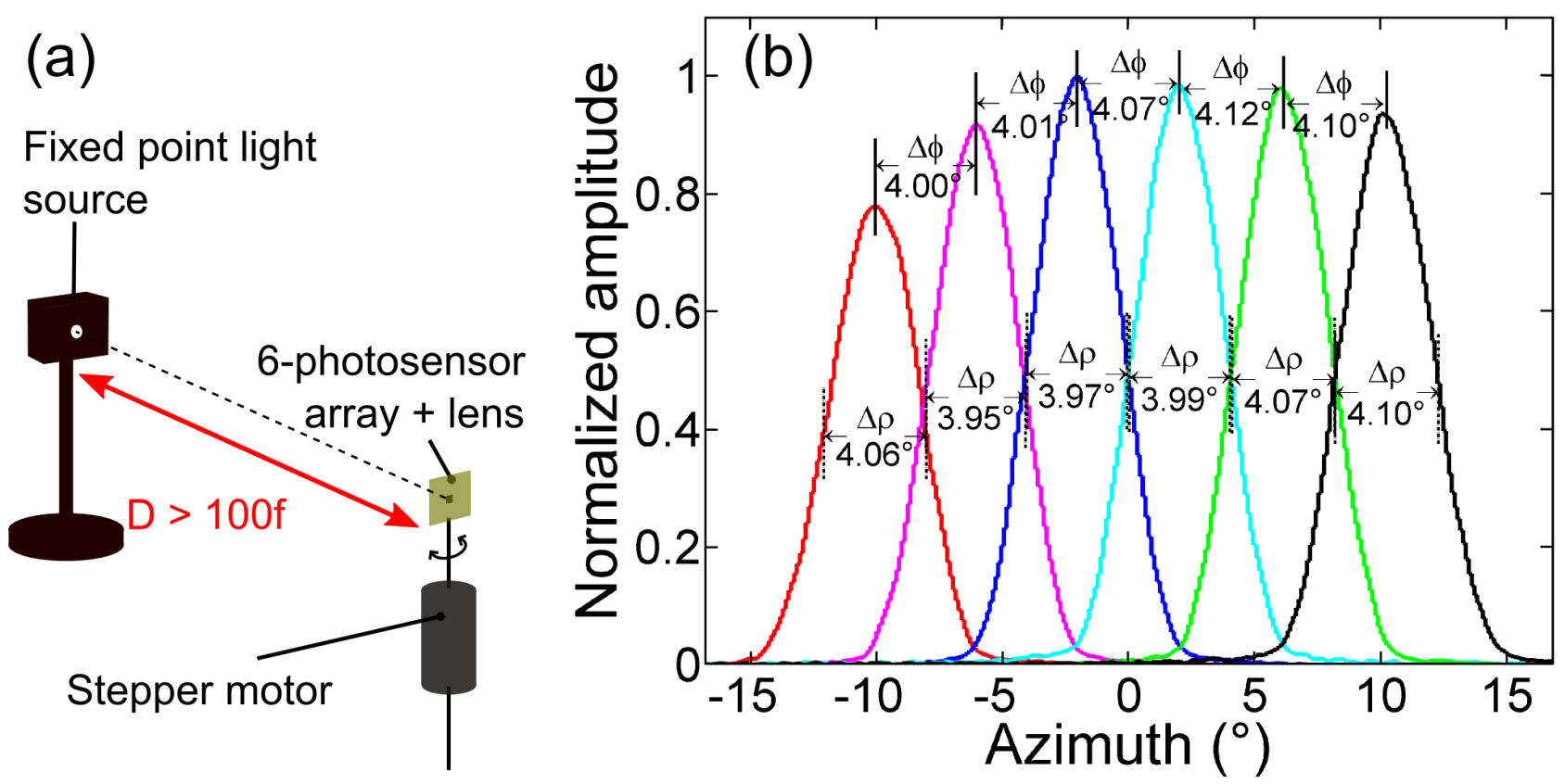

Fig. 2. (a) Scheme of the test bench used to determine the Gaussian ASFs of the 6-photosensor array obtained by slowly rotating the visual motion sensor mounted on the motor shaft of a stepper motor and placed at a distance $D=50 \mathrm{~cm}$ in front of a fixed point light source. (b) Raw Gaussian ASFs of the photosensor array.

bell-shaped ASF projected onto the photosensor array, as occurs in some diurnal insects [66], where:

$$
\Delta \varphi=\Delta \rho
$$

The visual photoreceptor axes are separated by an interreceptor angle $\Delta \varphi=4^{\circ}$ and each pixel features an acceptance angle $\Delta \rho=4^{\circ}$ [Fig. 2(b)]. The horizontal Field Of View (FOV) of the visual motion sensor is $28.8^{\circ}$.

2) Local Motion Sensor (LMS): Each LMS assesses the angular speed $\omega_{i}$ [i.e., a 1-D component of the OF, Fig. 3(a)] of any dark-to-light (ON) or light-to-dark (OFF) contrast in the same way as the fly's motion-detecting neurons. This "perceived" angular speed $\omega_{i}$ is transformed by the optical system into a delay $\Delta t_{i}$ between 2 neighboring photosensor signals defined as follows:

$$
\Delta t_{i}=\frac{\Delta \varphi}{\omega_{i}}
$$

The functional "time of travel" scheme used here consists of 6 processing steps [30], [54], [65] measuring the delay $\Delta t_{i}$, thus giving the angular speed $\omega_{i}^{m}$ (Fig. 3):

- Step 1: Low-pass spatial filtering is achieved by defocusing the lens, thus giving each pixel a Gaussian ASF.

- Step 2: Analog bandpass filtering: high-pass filtering $\left(f_{c}=20 \mathrm{~Hz}\right)$ enhances the contrast information and eliminates the DC component of the photoreceptor signals. This step is followed by a first-order low-pass filtering step, where $f_{c}=136 \mathrm{~Hz}$.

- Step 3: Digitizing and filtering: second-order fixed-point digital low-pass filtering $\left(f_{c}=30 \mathrm{~Hz}\right)$ reduces any high frequency noise introduced by the artificial indoor lighting $(100 \mathrm{~Hz})$.

- Step 4: Hysteresis thresholding is performed to distin- guish between 'ON' and 'OFF' contrast transitions (i.e. dark-to-light and light-to-dark transitions, respectively) in each channel.

- Step 5: A time delay circuit is triggered by one channel and stopped by the neighboring channel. This circuit measures the time $\Delta t_{i}$ elapsing between similar ('ON' or 'OFF') transitions occurring in two adjacent photoreceptors.

- Step 6: Computing the 1-D angular speed of a contrast in the visual field of the LMS, using a look-up table which converts the delay $\Delta t_{i}$ into the measured angular speed $\omega_{i}^{m}$.

3) Implementation and optimization: Our visual motion sensor generates 5 simultaneous local measurements $\omega_{i}^{m}$ of the 1-D angular speed of a moving natural panorama in a measurement range of more than one decade, ranging from $25 \% / s$ to $350 \%$. The sensor output is the median value $\omega_{\text {median }}^{m}$ of the 5 LMSs. The whole processing of the 5 LMSs and the computation of the median value were carried out on a dsPIC33FJ128GP802 $\mu C$ working at a sampling frequency of $2 \mathrm{kHz}$ and running at 40 MIPS. This lowpower 16-bit $\mu C$ was a very good candidate in term of size footprint (28 pins QFN-S package, see Table I for dimension), power consumption and performances allowing it to carry out the whole processing using a 16-bit MAC unit ("Multiplier + ACcumulation"), 1 SPI and $6 \times 12-$ bit ADCs (Analog to Digital Converters) while meeting with the constraints of MAVs [see Fig. 3(a)].

The $\mu C$ embedded onboard the visual motion sensor (Fig. 1) is connected to an external Bluetooth module via a testboard. This radio link allows the operator to record all the data synchronously and to convey it to a computer for analysis. The program of the 16-bit $\mu C$ was developed on 


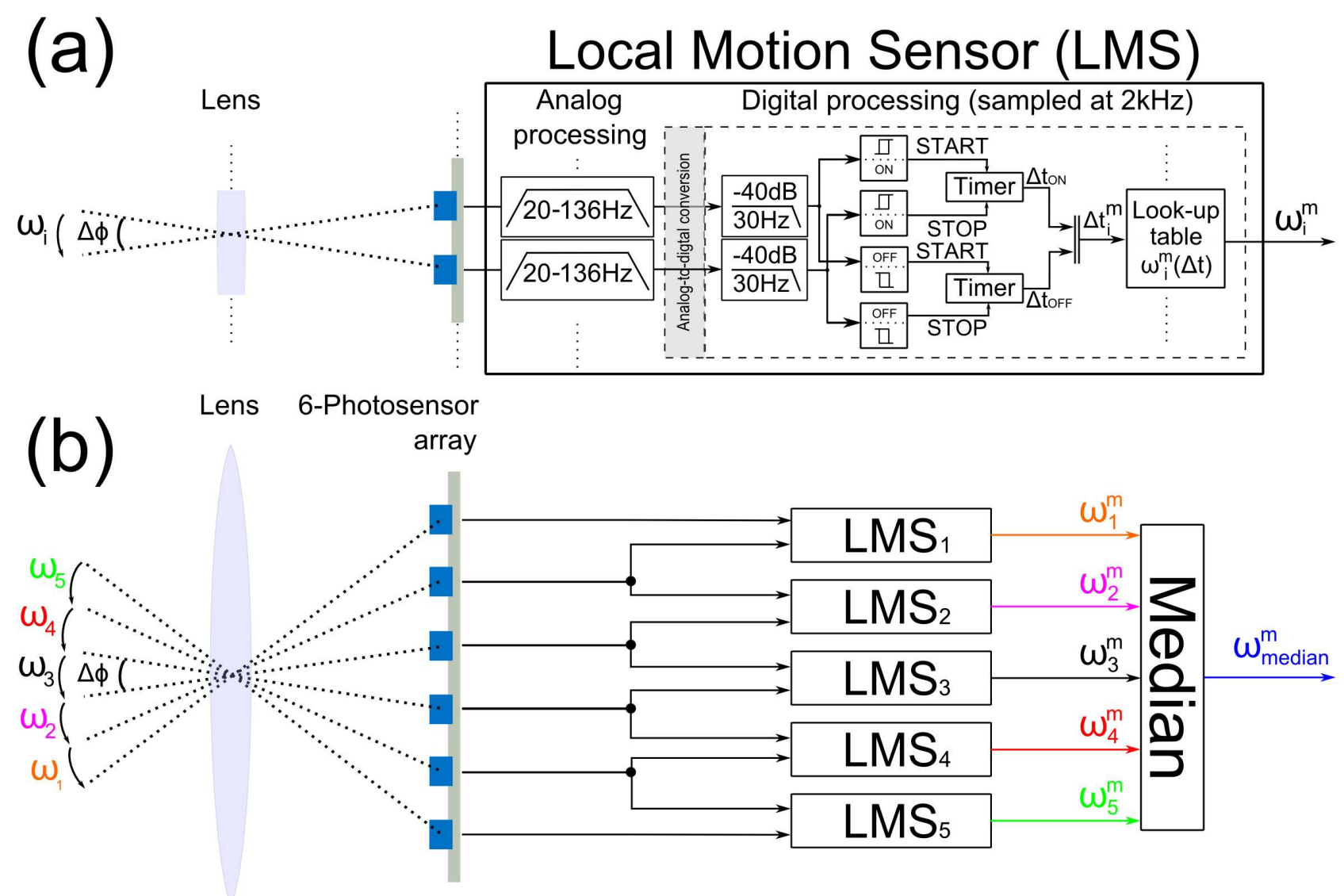

Fig. 3. General processing architecture of the visual motion sensor, including its 5 LMSs. (a) Processing architecture of one LMS. The visual signals delivered by neighboring photoreceptors are filtered both spatially and temporally by an analog bandpass filter with cutoff frequencies [20 $\mathrm{Hz}, 136 \mathrm{~Hz}]$ and a second order fixed-point digital low-pass filter with a cutoff frequency of $30 \mathrm{~Hz}$. The filtered photoreceptor signals are then thresholded to determine the angular speed $\omega_{i}^{m}$, using the "time of travel" scheme previously developed at our Laboratory [30], [31], [64], [65]. The "time of travel" $\Delta t_{i}$, which is proportional to the inverse of $\omega_{i}^{m}$, elapsing between two filtered photoreceptor signals is measured by a timer: $\Delta t_{O N}$ and $\Delta t_{O F F}$ are measured by means of ON and OFF contrast distinguishing processes [29]. These delays $\Delta t_{i}$ are used to generate the 1-D angular speed $\omega_{i}^{m}$ in the visual field of the 1-D LMS. (b) The 5 LMS output signals are combined to generate a more robust and frequently refreshed 1-D median measured angular speed $\omega_{\text {median }}^{m}$. The overall processing was carried out on a tiny low-power 16-bit $\mu \mathrm{C}$ at a sampling rate of $2 \mathrm{kHz}$.

Matlab/Simulink(c) environment and compiled using a specific toolbox (available on http://www.kerhuel.eu) developed for dsPIC $\mu C s$.

In order to be able to perform all the processing with the limited computational power of a tiny $\mu C$, several optimizations in the sensory fusion method were required to reduce the computational load. The order of the digital low-pass filter embedded in the $\mu C$ was reduced two-fold from the $4^{\text {th }}$ [54] to $2^{\text {nd }}$ order. This reduction was possible thanks to the onchip pre-amplification unit of the LSC retina, which reduces the noise. One simple 16-bit free counter was used to measure the 10 delays $\Delta t\left(\Delta t_{O N}\right.$ and $\Delta t_{O F F}$ of the $\left.5 \mathrm{LMSs}\right)$ required to estimate visual motion in the FOV of the 5 LMSs.

4) Characteristics of the visual motion sensor: The mass balance of our tiny device, including all the electronics, does not exceed 1 gram, which amounts to only $0.2 \mathrm{~g}$ per LMS (Table I). It is also a low-power visual motion sensor with a consumption of only $74 \mathrm{~mA}$. The specifications of the visual motion sensor are summarized in Table II.

We recenty showed that by using the same fusion algorithm, the measurement range of a similar sensor can be tuned to lower angular speeds by adjusting the optical parameters as
TABLE I

MASS BALANCE OF THE VISUAL MOTION SENSOR

\begin{tabular}{|c||c|}
\hline Parts & Mass $(\mathrm{g})$ \\
\hline PCB thickness $0.4 \mathrm{~mm}, 3 \mathrm{~cm}^{2}$ & 0.402 \\
\hline Lenslet Sparkfun SEN-00637 & 0.17 \\
\hline Lenslet-mount & 0.11 \\
\hline LSC iC-Haus retina & 0.13 \\
\hline$\mu C$ dsPIC $6 \times 6$ & 0.1 \\
\hline Electronic components & 0.162 \\
\hline Estimated total mass $=$ & 1.074 \\
\hline \multicolumn{2}{|c|}{ Real mass $=0.98 \mathrm{~g}$} \\
\hline \multicolumn{2}{|c|}{ Mass per 1-D LMS $<0.2 \mathrm{~g}$} \\
\hline
\end{tabular}

shown in [67].

\section{EXPERIMENT}

The visual motion sensor was tested indoors in natural light at a constant illuminance of approximately 1500lux, corresponding to the sunny daylight coming from a window. The visual motion sensor was placed at an orthogonal distance 
TABLE II

SPECIFICATIONS OF THE VISUAL MOTION SENSOR

\begin{tabular}{|c||c|}
\hline \multicolumn{1}{|c||}{ Retina } & LSC iC-Haus \\
\hline Inter-receptor angle $\Delta \varphi\left(^{\circ}\right)$ & 4 \\
\hline Acceptance angle $\Delta \rho\left(^{\circ}\right)$ & 4 \\
\hline Photodiode size $(\mu m)$ & $300 \times 1600$ \\
\hline Pixel pitch $(\mu m)$ & 420 \\
\hline Focal length of the lens $(m m)$ & 2 \\
\hline$F_{\text {number }}$ of the lens & 2.8 \\
\hline Angular velocity range $\left(^{\circ} / s\right)$ & {$[25 ; 350]$} \\
\hline Resolution $\left(^{\circ} / s\right)[$ Min; Max $]$ & {$\left[7 \times 10^{-2} ; 14.5\right]$} \\
\hline Sensitivity $\left(^{\circ} / s / L S B\right)$ & $7.63 \times 10^{-4}$ \\
\hline Measured mass with optics $(\mathrm{g})$ & $0.98 g$ \\
\hline
\end{tabular}

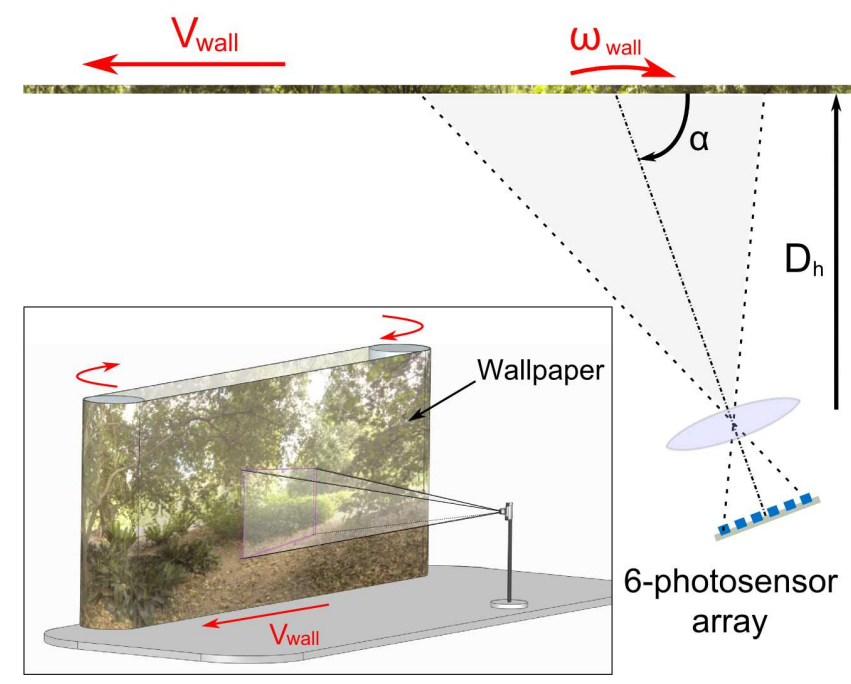

Fig. 4. Test bed used to assess the performances of the first sensory fusion method of the visual motion device based on a 6-pixel 1-D array. The visual motion sensor was placed at an orthogonal distance $D_{h}$ from a piece of wallpaper (forming a printed belt), at an arbitrary angle $\alpha$ between the direction of the wall motion $\left(\vec{V}_{\text {wall }}\right)$ and the main sensor axis. The printed belt depicting a natural colored panorama (inset) was stretched between two drums actuated thanks to a motor and a V-belt. The printed belt was made to move horizontally in a pre-determined preferred direction in front of the visual motion sensor at an angular speed $\omega_{\text {wall }}$.

$D_{h}$ from a printed belt of wallpaper showing a natural colored panorama. The printed band was stretched between 2 drums actuated thanks to a motor and a V-belt (see enclosed frame Fig. 4). The visual motion sensor was oriented at an arbitrary angle $\alpha$ between the direction of the wall motion $\left(\vec{V}_{\text {wall }}\right)$ and the main sensor axis (Fig. 4). The panorama was therefore made to move horizontally perpendicularly to the visual motion sensor which generated a ground truth optic flow seen by the sensor $\omega_{\text {wall }}$ and defined by (3):

$$
\omega_{\text {wall }}=\frac{V_{\text {wall }}}{D_{h}} \times \sin ^{2} \alpha
$$

By imposing an arbitrary orientation angle $\alpha$ to the visual motion sensor, we wanted to check if the measurements obtained with each $i^{\text {th }}$ LMS were in line with (4):

$$
\omega_{i}^{m}=\frac{V_{\text {wall }}}{D_{h}} \times \sin ^{2}(\alpha+(i-3) \times \Delta \varphi)
$$

The dynamic indoor responses were assessed by the visual motion sensor at $\alpha=60^{\circ}$ and $\alpha=80^{\circ}$ with 2 different printed belts:

- The first belt was decorated with a natural colored panorama [Fig. 5(m)],

- The second one was lined with a colored indoor panorama featuring a laboratory [Fig. 5(n)].

The wallpaper was moved using a triangular speed law involving a series of velocity ramps with various slopes ranging from $27 \% / s$ to $230^{\circ} / s$ with $\alpha=60^{\circ}$ and from $28^{\circ} / s$ to $312 \%$ with $\alpha=80^{\circ}$.

\section{RESUlTS}

The dynamic indoor responses of the visual motion sensor and the median output of the 5 LMSs were studied in terms of the refresh rate $\left(f_{\text {refresh }}\right)$ and the standard deviation error $\left(S t d_{\text {error }}\right)$ computed as follows:

$$
S t d_{\text {error }}=\operatorname{std}\left(\omega_{i}^{m}-\omega_{\text {wall }}\right)
$$

The $S t d_{\text {error }}$ therefore corresponds to the dispersion of the data between the measured angular speed $\omega_{i}^{m}$ and the groundtruth value $\omega_{\text {wall }}$. The main contributor is that of the 5 angular speed measurements $\omega_{i}^{m}$ which is most frequently used to calculate the median angular speed $\omega_{\text {median }}^{m}$. The refresh rate $\left(f_{\text {refresh }}\right)$ was defined as the number of new motion measurements per second. A new motion measurement occurs when a contrast transition is detected by one pixel and then by the second pixel with any delay $\Delta t$ in the angular speed measurement range [i.e. in the $25 \% / \mathrm{s}$ to $350 \% \mathrm{~s}$ range, see (2)].

As was to be expected in view of (4), the 5 LMS output measurements are different [Fig. 5(a), (d), (g) and (j)] because of the different orientations of the visual axes of the LMSs in the sensor's FOV. Figs. 5(c), (f), (i) and (l) show that the main contributors to the median value at the orientation angles $\alpha=60^{\circ}$ and $\alpha=80^{\circ}$ were the $3^{\text {rd }}$ LMS and the $5^{\text {th }}$ LMS, respectively. For both panoramas, the median value accurately followed the angular speed of the wall $\omega_{\text {wall }}$, giving a $S t d_{\text {error }}$ smaller than $12 \%$ in comparison with the value obtained with the main contributor, which was between $19 \%$ and $24 \%$. In addition, the refresh rate of the median value was found to increase more than 4-fold $(67 \mathrm{~Hz})$ in comparison with that observed in the case of the LMS main contributor (15.7 Hz) [Figs. 5(b), (e), (h) and (k)].

\section{ESTIMATION OF THE DIRECTION AND THE MAGNITUDE OF THE VISUAL MOTION}

\section{A. Device description}

The improved sensory fusion method of the new visual motion sensor presented in this section is based on the front end described in section II-1, having the optical characteristics described in Table II in terms of the inter-receptor angle $\Delta \varphi$ and the acceptance angle $\Delta \rho$. This visual motion sensor 

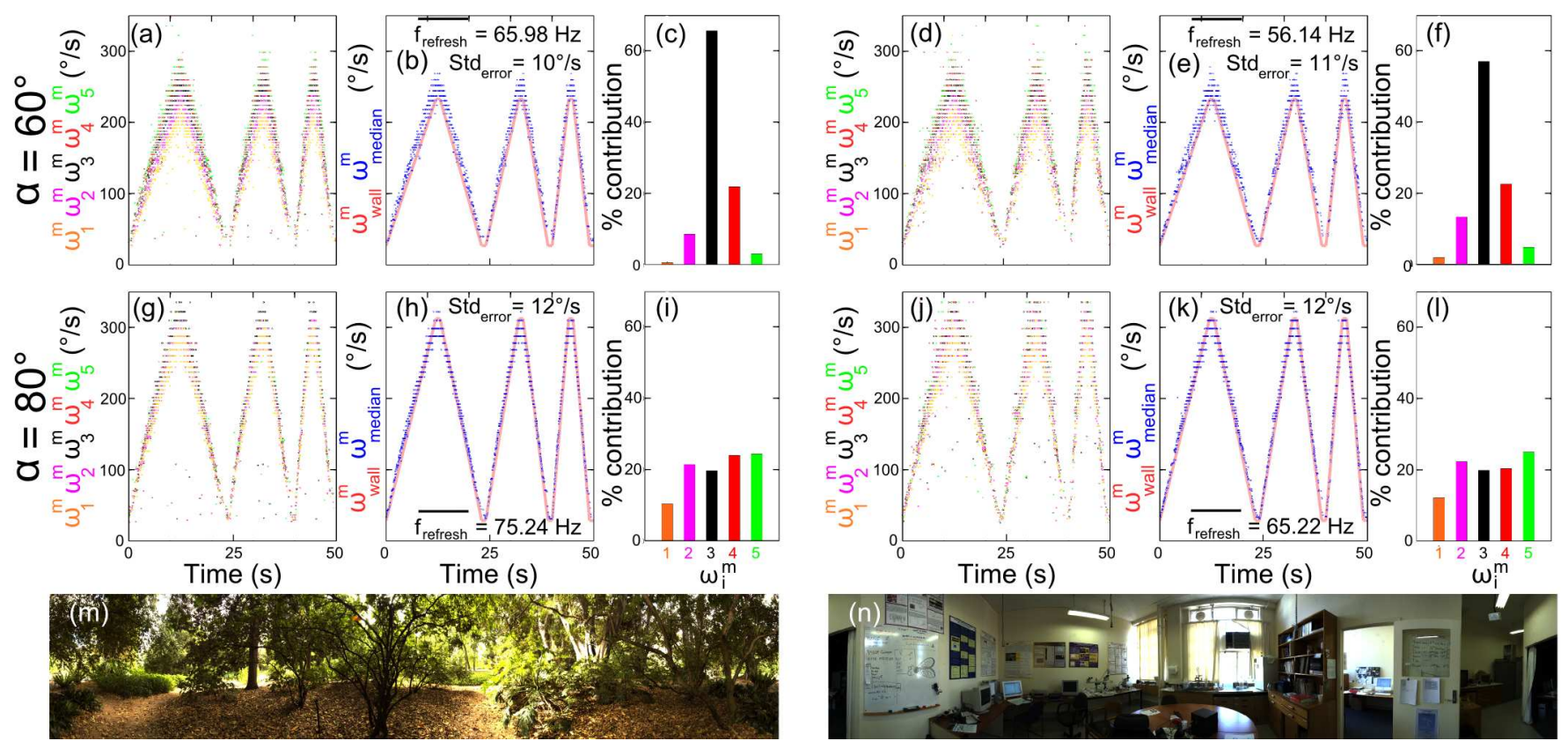

Fig. 5. Dynamic indoor responses of the visual motion sensor. The visual motion sensor was placed at an orthogonal distance $D_{h}=24 \mathrm{~cm}$ from a moving printed belt lined with a colored natural panorama depicting either bushes and trees or a laboratory. The visual motion sensor was placed at 2 different orientation angles $\alpha=60^{\circ}$ and $\alpha=80^{\circ}$ between the direction of the wall motion $\left(\vec{V}_{\text {wall }}\right)$ and the main sensor axis to check that each LMS measures visual motion in its own visual field [see (4)]. The printed belt was moved using a triangular law giving a triangular pattern of angular speed variations involving a series of velocity ramps with different slopes ranging from $27 \% / s$ to $230^{\circ} / s\left(\alpha=60^{\circ}\right.$ ) and from $28^{\circ} / s$ to $312^{\circ} / s\left(\alpha=80^{\circ}\right)$ [see (3)]. (a), (d), (g) and (j) Dynamic indoor responses of each LMS in the visual motion sensor placed at an orientation angle $\alpha=60^{\circ}\left[(\mathrm{a})\right.$ and (d)] and $\alpha=80^{\circ}[(\mathrm{g})$ and (j)]. Note that each LMS output differed from the others because of the different orientations of the LMS visual axes in the sensor's FOV as expected according to (4). (b), (e), (h), and (k) Dynamic indoor responses in terms of median values in comparison with those predicted by the main contributor, along with the standard deviation error $\left(S t d_{\text {error }}\right)$ and refresh rate $\left(f_{\text {refresh }}\right)$ characteristics. (c), (f), (i), and (1) Vertical bar graph showing which LMS in the visual motion sensor was the main contributor to the median value computed. $(\mathrm{m})$ and $(\mathrm{n})$ The natural colored panorama depicted on the printed belt (Fig. 4) used to assess the visual motion sensor's performances.

is able to estimate the direction and the magnitude of the visual motion $\omega_{\text {median }}^{\max }$ on the basis of 10 angular speed measurements: 5 LMSs are used to compute the median angular speed $\omega_{\text {median }+/-}^{m}$ in each direction of motion ("+" or "-") (Fig. 6). In order to determine the direction of the visual motion without any prior knowledge, empirical findings [38] have shown that, within a given angular speed range, the angular speed of the contrasts detected in the correct motion direction is usually greater than that measured in the opposite direction. Based on this finding, by simply choosing the maximum value of the median angular speeds in the two directions $\omega_{\text {median }}^{m}$ and $\omega_{\text {median-, }}^{m}$, it is possible to determine the direction of the visual motion accurately in the $\left[-350^{\circ} / s ;-80^{\circ} / s\right] \cup\left[80^{\circ} / s ; 350^{\circ} / s\right]$ range. The ] $-80^{\circ} / s ; 80 \% / s$ [ range corresponds to an uncertainty range, where the direction and the magnitude of the angular speed cannot be assessed accurately. As soon as the sensor detects visual motion in the $]-80^{\circ} / s ; 80 \% / s$ [ range, the output signal $\omega_{\text {median }}^{\max }$ magnitude and direction are voluntary set to "no value" without any error.

\section{B. Optimization of the motion direction estimates}

To optimize the motion direction estimation, we decided to filter each median angular speed measurement $\left(\omega_{\text {median }}^{m}\right.$ and $\omega_{\text {median_- }}^{m}$ ) using a rate-limiter that removes any value that is too different from the previous angular speed measurement knowing the $\mathrm{OF}$ rate is bounded. A sliding window filters out any motion direction error by selecting the direction occurring more than 8 times among the last 16 detected motion directions. Thanks to this filtering process, the motion direction was perfectly determined (Fig. 6).

The improved sensory fusion method was optimized in order to increase the number of LMSs embedded into the same $\mu C$ two-fold. The same filtered visual signals were recombined in order to compute an accurate visual angular speed and the direction of the visual motion while keeping the digital processing frequency at $2 \mathrm{kHz}$. The median computation step was optimized by computing the median value only whenever a new visual motion measurement occurred, i.e., whenever a new $i^{\text {th }}$ LMS angular speed $\omega_{i+/-}^{m}$ was measured: this algorithm optimization prevents the $\mu C$ from being overloaded by computing the median value at all the time steps at which none of the LMS outputs are refreshed.

All these improvements have made the tiny $\mu C$ capable of carrying out all the processing operations required to determine the median 1-D angular speed of a natural panorama $\omega_{\text {median }}^{\max }$ and to estimate the direction of motion with a mean computational load of only $53 \%$ (minimum: $43 \%$; maximum: $82 \%$-very short peaks-) at a sample frequency of $2 \mathrm{kHz}$.

\section{Experiment}

The static and dynamic responses of the improved sensory fusion method of the visual motion sensor presented here were obtained under 2 lighting conditions. The background 


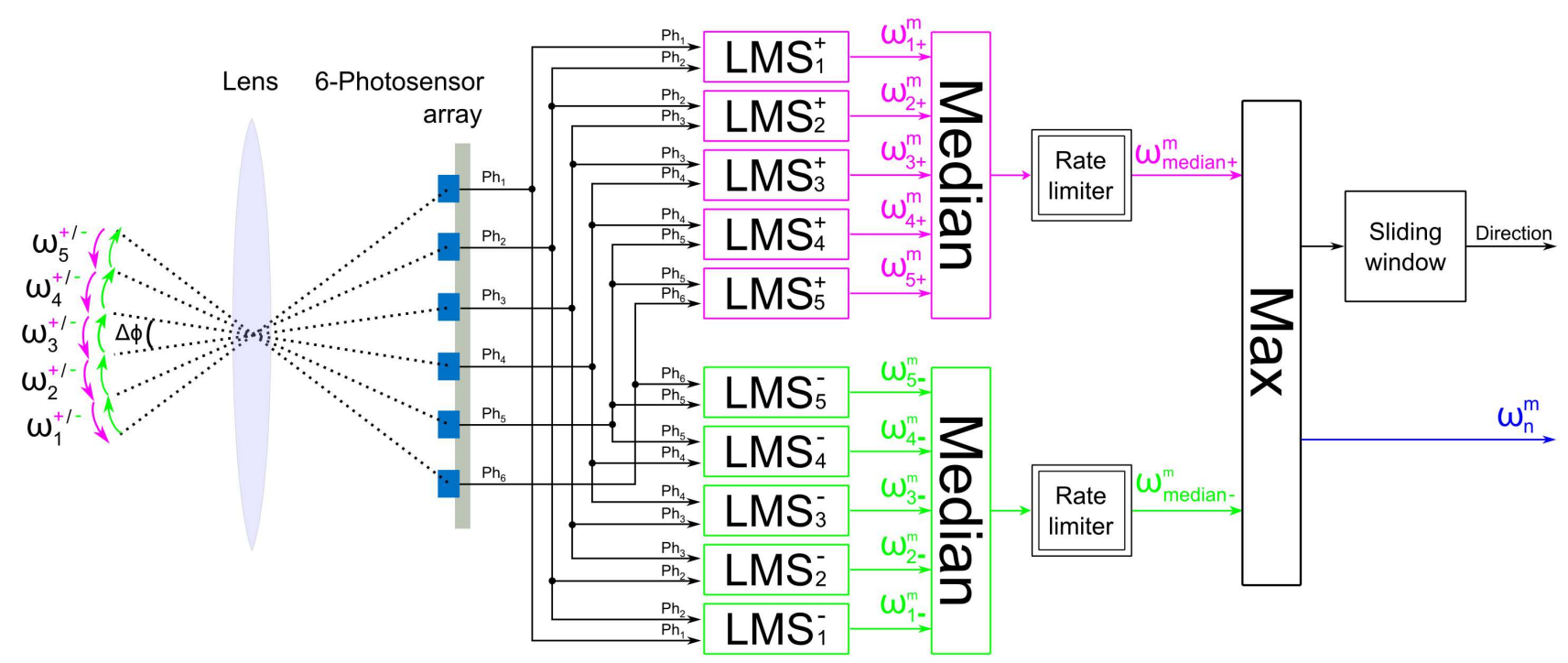

Fig. 6. General processing architecture of the improved sensory fusion method based on 10 LMSs. The visual signals delivered by the photoreceptors are filtered and thresholded by the LMSs to determine the angular speeds $\omega_{i+/-}^{m}$ using the "time of travel" scheme in the two directions of motion [30], [31], [38], [65]. The visual motion is measured in the opposite direction by reversing the inputs to each LMS. A rate limiter function filters out any median angular speed measurement that changes too fast. The motion direction and magnitude $\omega_{\text {max }}^{\max }$ are estimated based on a simple algorithm, using the maximum median value of the angular speed $\omega_{\text {median }}^{m}$ and $\omega_{\text {median- }}^{m}$ computed from the 5 LMSs in the 2 directions of motion. A sliding window removes any motion direction error by selecting the direction occurring more than 8 times among the last 16 detected motion directions. This improved sensory fusion method allows to measure the motion magnitude efficiently in the $\left[-350^{\circ} / \mathrm{s} ;-80 \% / \mathrm{s}\right] \cup[80 \% / s ; 350 \% / \mathrm{s}]$ range and to determine the direction of motion without any prior knowledge.

irradiance values were measured in $W . \mathrm{cm}^{-2}$ using a digital radiometer (ILT1700) which gives the irradiance in the direction of the radiometer's sensor. The visual motion sensor was placed at an orthogonal distance $D_{h}=24 \mathrm{~cm}$ from a printed belt, oriented at an angle $\alpha=90^{\circ}$. The printed belt was stretched between 2 drums actuated by a motor and a V-belt which could be made to rotate either clockwise or anticlockwise (see inset in Fig. 7). The panorama was therefore made to move horizontally in two directions with respect to the visual motion sensor at an angular speed $\omega_{\text {wall }}$ according to (3). The static responses of the visual motion sensor were assessed by applying a series of $30 \% \mathrm{~s}$ fifteensecond steps to the moving wall at a rotational speed $\omega_{\text {wall }}$ in the $[-315 \% / s ;-105 \% / s] \cup[105 \% / s ; 315 \% / s]$ range in the two opposite directions. These experiments were conducted with an irradiance of $5 \times 10^{-3}$ W.cm ${ }^{-2}$.

The dynamic characteristics of the visual motion sensor were assessed at two different illuminance values: at $2.5 \times 10^{-2} W . \mathrm{cm}^{-2}$, which corresponds to strong sunlight coming from a windows and $5 \times 10^{-3} \mathrm{~W} \cdot \mathrm{cm}^{-2}$, which corresponds to strong indoor lighting. We applied a 60 -second stimulus to the moving wall, involving a series of velocity ramps with different slopes in the $[-300 \% / s ; 300 \% / s]$ range. The belt was covered with a natural colored panorama showing bushes and trees [Fig. 8(g)] or with a colored indoor panorama featuring a laboratory [Fig. 8(h)].

\section{Results}

To assess the static characteristics of the visual motion sensor, we studied the mean standard deviation of the data,

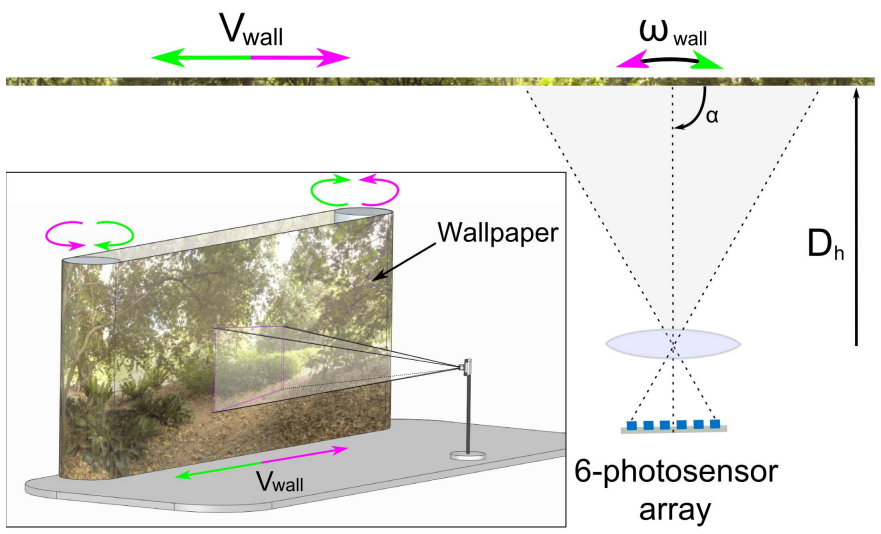

Fig. 7. Test bed used to assess the performances of the visual motion device including the $10 \mathrm{LMSs}$ and the motion direction detection unit. The visual motion sensor was placed at an orthogonal distance $D_{h}=24 \mathrm{~cm}$ from a printed belt. In this case, the angle $\alpha$ between the direction of the wall motion $\left(\vec{V}_{\text {wall }}\right)$ and the main sensor axis was $\alpha=90^{\circ}$. The belt printed with a natural colored panorama depicting either bushes and trees or a laboratory, was stretched between two drums actuated by a motor and a V-belt: the belt could be made in this case to rotate either clockwise or anticlockwise. The panorama was therefore made to move horizontally in either direction.

which was computed as follows:

$$
\overline{S t d}=\overline{\operatorname{std}\left(\omega_{\text {median }}^{\max }\right)}
$$

The best linear approximation was computed to determine the accuracy of our visual motion sensor. This criterion was calculated on the basis of (7):

$$
\omega_{\text {median }}^{\max }=a \times \omega_{\text {wall }}
$$


where $\omega_{\text {median }}^{\max }$ is the output signal of the visual motion sensor and $\omega_{\text {wall }}$ is the angular speed of the moving wall as seen by the visual motion sensor. The regression coefficient $a$ of (7) was used to compute the linearity error given by (8):

$$
\text { Linearity } \operatorname{Error}(\%)=|(a-1)| \times 100
$$

The dynamic responses of the median output $\omega_{\text {median }}^{\max }$ of the tiny visual motion sensor were assessed in comparison with the perceived angular speed $\omega_{\text {wall }}$ in terms of the refresh rate and the $S t d_{\text {error }}$ defined in (5).

1) Static characteristics: Figs. 8(a) and (b) show the static characteristics of the visual motion sensor tested indoors in front of a moving wall at an irradiance value of $5 \times 10^{-3} W \cdot \mathrm{cm}^{-2}$. As shown in Figs. 8(a) and (b), the visual motion sensor responded accurately with a really low LinearityError rate [see (8)] of less than $1 \%$ and an excellent dispersion of less than $7 \% \mathrm{~s}$. In the measurement range of $\left[-350^{\circ} / s ;-80^{\circ} / s\right] \cup\left[80^{\circ} / s ; 350^{\circ} / s\right]$, the visual motion sensor estimated the direction of motion perfectly without making a single direction error.

2) Dynamic characteristics: Figs. 8(c) and (d) show the dynamic responses of the visual motion sensor at an irradiance of $2.5 \times 10^{-2} W . \mathrm{cm}^{-2}$. The median value closely obeyed the triangular law imposed on the angular speed of the wall $\omega_{\text {wall }}$, giving a low $S t d_{\text {error }}$ of only $7.4 \% / s$ and $6.23 \% / s$ with the outdoor and indoor panoramas, respectively. At a lower irradiance of $5 \times 10^{-3} \mathrm{~W} . \mathrm{cm}^{-2}$, the median value $\omega_{\text {median }}^{\max }$ again closely obeyed the triangular law imposed on the angular speed of the moving wall $\omega_{\text {wall }}$, with a $S t d_{\text {error }}$ of $9.2 \% \mathrm{~s}$ in the case of the bushes and trees panorama and $5.44 \% / \mathrm{s}$ in that of the laboratory panorama. Despite the difference in the irradiance, the $S t d_{\text {error }}$ was always of a similar order of magnitude. In any case, the visual motion sensor gave a highly refreshed output. As was to be expected from [57], the $f_{\text {refresh }}$ increased with the irradiance, amounting to $50.6 \mathrm{~Hz}$ at an irradiance of $5 \times 10^{-3} W . \mathrm{cm}^{-2}$ and $74.5 \mathrm{~Hz}$ at a higher value of $2.5 \times 10^{-2} W . \mathrm{cm}^{-2}$ when the outdoor panorama was displayed on the printed belt [Fig. 8(c) and (e)]. Similar results were obtained with the indoor panorama: $f_{\text {refresh }}$ of $39.7 \mathrm{~Hz}$ at $5 \times 10^{-3} W . \mathrm{cm}^{-2}$ and $62.1 \mathrm{~Hz}$ at $2.5 \times 10^{-2} W . \mathrm{cm}^{-2}$ [Fig. $8(\mathrm{~d})$ and (f)]. The motion direction was estimated perfectly by the sensor without making a single error.

In view of these performances, this novel tiny visual motion sensor can be said to provide a remarkably promising tool for performing robotic tasks such as obstacle avoidance and terrain following in forward flight, while meeting the requirements in very low avionic payload, since the total mass balance of the two-directional visual motion sensor does not exceed $1 \mathrm{~g}$.

\section{CONCLUSION}

In this study, two different sensory fusion methods of a 1-gram insect-inspired visual motion sensor were evaluated indoors under two different lighting conditions. The dynamic and static responses of this novel fly-inspired visual motion sensor were used to assess the performances of these very lightweight, low-power sensors, which can be mounted onboard tomorrow's MAVs for obstacle avoidance and speed control purposes.

The first sensory fusion method of our 1-gram $\mu C$-based visual motion sensor, consisting of a 5-LMS array, gave 5 simultaneous angular speed measurements and a single combined output in the $\left[25^{\circ} / s ; 350^{\circ} / \mathrm{s}\right]$ range, in a single preferred direction of visual motion. The results obtained in the study (Fig. 5) show how the accuracy and the robustness of the angular speed measurement have been improved thanks to our simple method of data combination based on the median operator. This method improves the $S t d_{\text {error }}$ more than 1.7fold from $19 \% / s$ in the case of the main contributor to the median value to $11 \%$ in the case of the median angular speed $\omega_{\text {median. }}^{m}$. The refresh rate of the visual motion sensor was found to have increased at least 4-fold $(67 \mathrm{~Hz})$ in comparison with that of the main contributor $(15.7 \mathrm{~Hz})$.

The excellent performances obtained with the first sensory fusion method of this 1-gram fly-inspired visual motion sensor led us to design an improved sensory fusion method incorporated into our visual sensor based on the same electronics. These improvements allowed to determine the direction and magnitude $\omega_{\text {median }}^{\max }$ of visual motion without any prior knowledge by recombining the filtered visual signals and processing the "time of travel" in the two opposite directions. This improved sensory fusion method of the 1-gram $\mu C$-based visual motion sensor designed and built at our Laboratory is based on a 10 LMS-array which can measure the direction and the magnitude of motion in the $\left[-350^{\circ} / s ;-80^{\circ} / s\right] \cup$ $\left[80^{\circ} / s ; 350 \% / s\right]$ range, thanks to the maximum operator value computed between the median angular speed in the two directions of motion ( $\omega_{\text {median }}^{m}$ and $\left.\omega_{\text {median- }}^{m}\right)$. The dynamic and static characteristics of this novel sensor (Fig. 8) were used to assess its performances. It consistently measured the 1-D angular speed accurately with an excellent LinearityError $<1 \%$. The impressive results obtained indoors were robust since the $S t d_{\text {error }}$ was of the same order of magnitude (less than $10^{\circ} / \mathrm{s}$ ) under two different irradiance conditions, whether the printed belt simulating an unknown environment depicted a natural landscape or a laboratory. Due to the size of the setup we used, the performances have been assessed only indoors. Nevertheless, we have shown recently in [57] that a very similar visual motion sensor based on the same retina could robustly and accurately measure the OF indoors and outdoors in a 1.5-decade illuminance range with strong transient variations.

This stand-alone sensor weighs less than $1 g$. The outstanding performances of this tiny $\mu C$-based visual motion sensor show that it constitutes a good trade-off between the need for reliable motion sensors and the limited power and avionic payload available on MAVs. This $1 \mathrm{~g}$ two-directional visual motion sensor yields at its output an accurate and highly refreshed angular speed measurement in the range of $\left[-350^{\circ} / s ;-80^{\circ} / s\right] \cup\left[80^{\circ} / s ; 350^{\circ} / s\right]$ perfectly adapted to any MAV flying forward and performing robotic tasks such as obstacle avoidance, terrain following, take-off, landing and speed-control purposes in forward flights even possibly for lunar landers [68].

In future works an other optimization of the "time of travel", called the interpolation-based "time of travel" scheme [60] can 

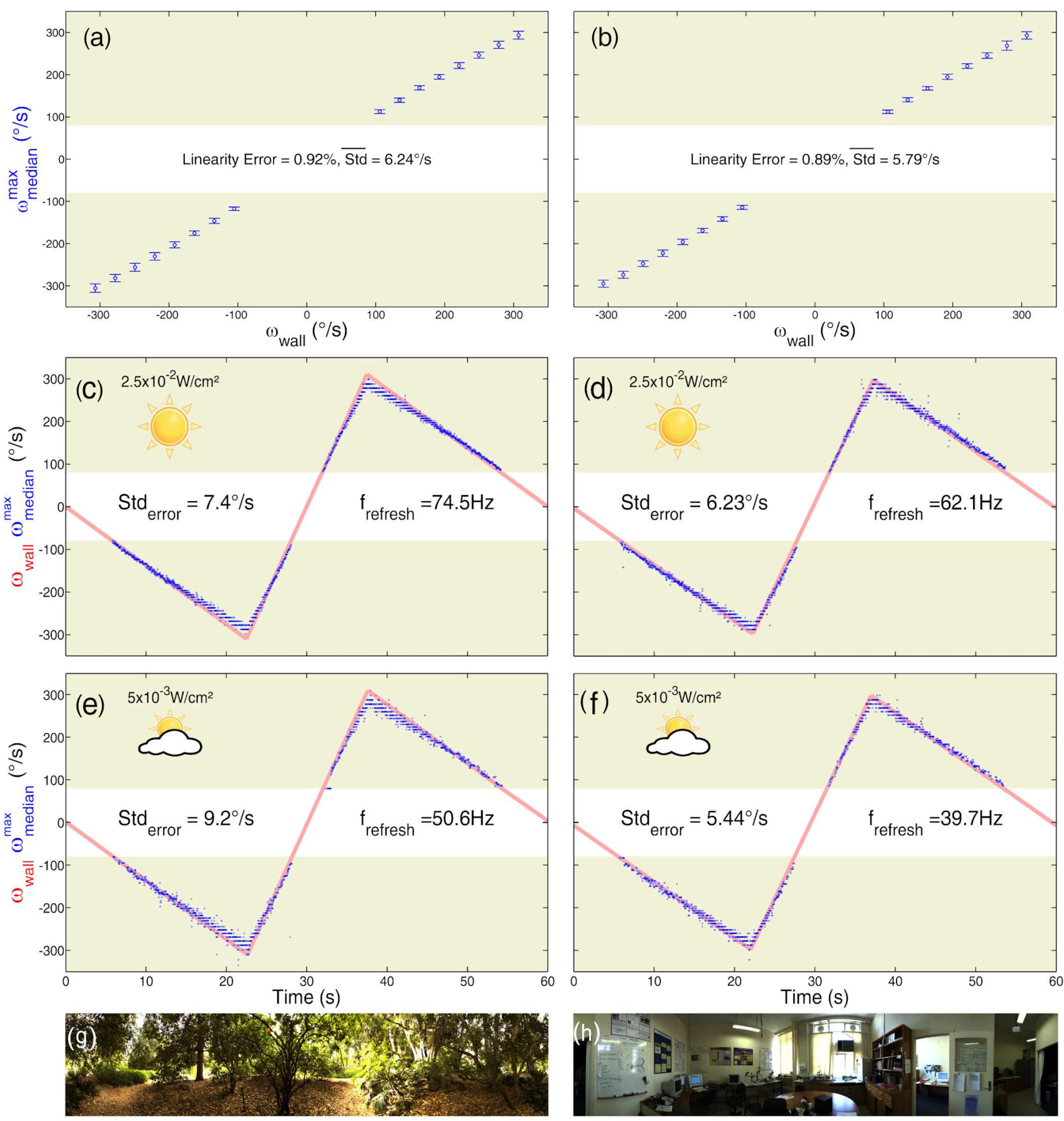

Fig. 8. Dynamic and static indoor responses of the visual motion sensor placed at an orthogonal distance $D_{h}=24 \mathrm{~cm}$ from the moving wall at an angle $\alpha=90^{\circ}$. The static indoor characteristics of the visual motion sensor were assessed by applying $30 \% / s$ steps (lasting $15 \mathrm{~s}$ ) to the printed belt in the $[-315 \% / s ;-105 \% / s] \cup\left[105^{\circ} / s ; 315 \% / s\right]$ range. The mean visual motion recorded at each angular speed $\omega_{w a l l}$ is plotted in the figure with its standard deviation. The best linear approximation obtained in each experiment was computed, and the departure from linearity is given as a percentage. The dynamic responses of the visual motion sensor were assessed at two different irradiance values of $5 \times 10^{-3} \mathrm{~W} \cdot \mathrm{cm}^{-2}$ and $2.5 \times 10^{-2} \mathrm{~W} \cdot \mathrm{cm}-2$ with the two printed panoramas. The printed belt was moved using a triangular law giving a triangular pattern of angular speed variation involving a series of velocity ramps ranging from $-300 \% / \mathrm{s}$ to $300 \% \mathrm{~s}$. A fusion algorithm based on the maximum median value of the two opposite directions was used to determine the magnitude $\omega_{\text {median }}^{\max }$ and the direction of the angular speed. (a) and (b) Static indoor characteristics of the visual motion sensor. With both panoramas, the visual motion sensor yielded accurate median angular speed measurements with only a small LinearityError of less than $1 \%$ and an excellent $\overline{S t d}$ of less than $7 \% / \mathrm{s}$. (c)-(f) Dynamic indoor responses of the median angular speed $\omega_{\text {max }}^{\max }$ of the visual motion sensor, along with the standard deviation error $\left(S t d_{\text {error }}\right)$ and refresh-rate $\left(f_{\text {refresh }}\right)$ data. With the printed belt depicting bushes and trees [Fig. 8(c) and (e)], the results showed a small dispersion of less than $10 \% \mathrm{~s}$ and the refresh rate increased from $50.6 \mathrm{~Hz}$ to $74.5 \mathrm{~Hz}$ with the irradiance. With the printed belt depicting a laboratory, the results show that the dispersion was less than $7^{\circ} / \mathrm{s}$, and the refresh rate again increased with the irradiance from $39.7 \mathrm{~Hz}$ to $62.1 \mathrm{~Hz}$. (g) and (h) The natural colored panorama depicted on the printed belt (Fig. 7) used to assess the visual motion sensor's performances. 
be used to implement a larger number of 2-pixel LMS into a single dsPIC $\mu C$ and therefore process the OF from a much larger 2D retina.

\section{ACKNOWLEDGMENT}

We thank the anonymous referees. We are grateful to S. Viollet and N. Franceschini for their fruitful comments and suggestions during this study. We thank J. Blanc for correcting the English manuscript and R. Brinkworth and D. O'Carroll (Adelaide Uni., Australia) for kindly making their High Dynamic Range panoramic images available to us. We are most grateful to J. Diperi, D. Dray and Y. Luparini for their involvement in the mechanical design of the test bed and to M. Boyron and B. Fuschlock for their involvement in the overall electronic design of the test board and the visual motion sensor.

\section{REFERENCES}

[1] F. Iida, "Goal-directed navigation of an autonomous flying robot using biologically inspired cheap vision," in Proceedings of the 32nd International Symposium on Robotics (ISR), vol. 21, Seoul, South Korea, April 2001, pp. 1404-1409.

[2] W. Green, P. Oh, and G. Barrows, "Flying insect inspired vision for autonomous aerial robot maneuvers in near-earth environments," in IEEE International Conference on Robotics and Automation (ICRA), vol. 3, New-Orleans, USA, April 2004, pp. 2347-2352.

[3] J. C. Zufferey and D. Floreano, "Fly-inspired visual steering of ultralight indoor aircraft," IEEE Transactions on Robotics, vol. 22(1), pp. 137-146, 2006.

[4] F. Ruffier and N. Franceschini, "Aerial robot piloted in steep relief by optic flow sensors," in Proceedings of the IEEE/RSJ International Conference on Intelligent Robots and Systems (IROS), Nice, France, September 2008, pp. 1266-1273.

[5] A. Beyeler, J.-C. Zufferey, and D. Floreano, "optiPilot: control of takeoff and landing using optic flow," in European Micro Aerial Vehicle Conference (EMAV), vol. 27, Delft, Nederlands, September 2009, pp. 201-219.

[6] J. Humbert, J. K. Conroy, C. Neely, and G. Barrows, "Widefield integration methods for visuomotor control," in Flying insects and robots, D. Floreano, J. C. Zufferey, M. Srinivasan, and C. Ellington, Eds. Berlin: Springer Berlin Heidelberg, 2009, pp. 63-71.

[7] G. de Croon, E. de Weerdt, C. de Wagter, B. Remes, and R. Ruijsink, "The appearance variation cue for obstacle avoidance," IEEE Transactions on Robotics, vol. 28(2), pp. 529-534, 2012.

[8] F. Ruffier and N. Franceschini, "Octave, a bioinspired visuo-motor control system for the guidance of micro-air vehicles," in SPIE Conference on Bioengineered and Bioinspired Systems, A. D. Rodriguez-Vazquez, A. and R. Carmona, Eds., vol. 5119, Maspalomas, Spain, May 2003, pp. $1-12$.

[9] - "Optic flow regulation: the key to aircraft automatic guidance," Robotics and Autonomous Systems, vol. 50(4), pp. 177-194, 2005.

[10] N. Franceschini, F. Ruffier, and J. Serres, "A bio-inspired flying robot sheds light on insect piloting abilities," Current Biology, vol. 17(4), pp. 329-335, 2007.

[11] J. Zufferey, A. Beyeler, and D. Floreano, "Autonomous flight at low altitude using light sensors and little computational power," International Journal of Micro Air Vehicles, vol. 2(2), pp. 107-117, 2010.

[12] B. Herisse, T. Hamel, R. Mahony, and F.-X. Russotto, "Landing a VTOL Unmanned Aerial Vehicle on a moving platform using optical flow," IEEE Transactions on Robotics, vol. 28(1), pp. 77-89, 2012.

[13] F. Kendoul, I. Fantoni, and K. Nonami, "Optic flow-based vision system for autonomous 3D localization and control of small aerial vehicles," Robotics and Autonomous Systems, vol. 57(6-7), pp. 591-602, 2009.

[14] L. Kerhuel, S. Viollet, and N. Franceschini, "Steering by gazing: an efficient biomimetic control strategy for visually guided micro aerial vehicles," IEEE Transactions on Robotics, vol. 26(2), pp. 307-319, 2010.

[15] F. L. Roubieu, J. Serres, N. Franceschini, F. Ruffier, and S. Viollet, "A fully-autonomous hovercraft inspired by bees; wall-following and speed control in straight and tapered corridors," in IEEE International Conference on Robotics and Biomimetics (ROBIO), Guangzhou, China, December 2012 (in press).
[16] T. Collett, "Some operating rules for the optomotor system of a hoverfly during voluntary flight," Journal of Comparative Physiology A: Neuroethology, Sensory, Neural, and Behavioral Physiology, vol. 138(3), pp. 271-282, 1980.

[17] H. Wagner, "Flow-field variables trigger landing in flies," Nature, vol. 297(5862), pp. 147-148, 1982.

[18] M. Srinivasan, S. Zhang, M. Lehrer, and T. Collett, "Honeybee navigation en route to the goal: visual flight control and odometry," Journal of Experimental Biology, vol. 199(1), pp. 237-244, 1996.

[19] L. F. Tammero and M. H. Dickinson, "The influence of visual landscape on the free flight behavior of the fruit fly drosophila melanogaster," Journal of Experimental Biology, vol. 205(3), pp. 327-343, 2002.

[20] E. Baird, M. Srinivasan, S. Zhang, R. Lamont, and A. Cowling, "Visual control of flight speed and height in the honeybee," in From Animals to Animats 9, S. Nolfi, G. Baldassarre, R. Calabretta, J. Hallam, D. Marocco, J.-A. Meyer, O. Miglino, and D. Parisi, Eds. Springer Berlin / Heidelberg, 2006, vol. 4095, pp. 40-51.

[21] J. Serres, G. Masson, F. Ruffier, and N. Franceschini, "A bee in the corridor: centering and wall-following," Naturwissenschaften, vol. 95(12), pp. 1181-1187, 2008.

[22] E. Baird, T. Kornfeldt, and M. Dacke, "Minimum viewing angle for visually guided ground speed control in bumblebees," The Journal of Experimental Biology, vol. 213, pp. 1625-1632, 2010.

[23] A. Straw, S. Lee, and M. Dickinson, "Visual control of altitude in flying Drosophila," Current Biology, vol. 20(17), pp. 1550-1556, 2010.

[24] G. Portelli, F. L. Roubieu, F. Ruffier, and N. Franceschini, "Honeybees' speed depends on dorsal as well as lateral, ventral and frontal optic flows," PLOS ONE, vol. 6(5), p. e19486, 2011.

[25] M. Srinivasan, "Honeybees as a model for the study of visually guided flight, navigation, and biologically inspired robotics," Physiological Reviews, vol. 91(2), pp. 413-460, 2011.

[26] J. Gibson, The perception of the visual world. New York: Houghton Mifflin, 1950.

[27] K. Hausen, "The lobula-complex of the fly: structure, function and significance in visual behaviour," in Photoreception and Vision in Invertebrates, M. Ali., Ed. New York: Plenum, 1984, pp. 523-559.

[28] N. Franceschini, "Early processing of colour and motion in a mosaic visual system," Neuroscience Research (Suppl. 2), pp. 17-49, 1985.

[29] N. Franceschini, A. Riehle, and A. Le Nestour, "Directionally selective motion detection by insect neurons," in Facets of vision, D. Stavanga and R. Hardie, Eds. Berlin: Springer-Verlag, 1989, pp. 360-390.

[30] C. Blanes, "Appareil visuel élémentaire pour la navigation à vue d'un robot mobile autonome (Adviser: N. Franceschini)," Master's thesis, 1986.

[31] J. Pichon, C. Blanes, and N. Franceschini, "Visual guidance of a mobile robot equipped with a network of self-motion sensors," in Proceedings of SPIE: Mobile Robots IV, W. J. Wolfe and W. H. C. Editors, Eds., vol. 1195. Bellingham, USA: Society of Photo-optical Instrumentation Engineers, 1989, pp. 44-53.

[32] N. Martin and N. Franceschini, "Obstacle avoidance and speed control in a mobile vehicle equipped with a compound eye," in Proceedings of the Intelligent Vehicles Symposium (IV), Paris, France, October 1994, pp. 381-386.

[33] J. Serres, D. Dray, F. Ruffier, and N. Franceschini, "A vision-based autopilot for a miniature air vehicle: joint speed control and lateral obstacle avoidance," Autonomous Robot, vol. 25(1), pp. 103-122, 2008.

[34] G. Portelli, J. Serres, F. Ruffier, and N. Franceschini, "Modelling honeybee visual guidance in a 3D environment," Journal of PhysiologyParis, vol. 104(1-2), pp. 27-39, 2010.

[35] N. Franceschini, J. M. Pichon, and C. Blanes, "From insect vision to robot vision," Philosophical Transactions of the Royal Society B: Biological Sciences, vol. 337, pp. 283-294, 1992.

[36] T. Netter and N. Franceschini, "A robotic aircraft that follows terrain using a neuromorphic eye," in IEEE/RSJ International Conference on Intelligent Robots and Systems (IROS), Lausanne, Switzerland, September 2002, pp. 129-134.

[37] F. Expert and F. Ruffier, "Controlling docking, altitude and speed in a circular high-roofed tunnel thanks to the optic flow," in IEEE/RSJ International Conference on Intelligent Robots and Systems (IROS), Vilamoura, Portugal, October 2012 (in press), pp. 1125-1132.

[38] C. Blanes, "Guidage visuel d'un robot mobile autonome d'inspiration bionique (Adviser: N. Franceschini)," Ph.D. dissertation, INP Grenoble, 1991.

[39] J. Plett, A. Bahl, M. Buss, K. Khnlenz, and A. Borst, "Bio-inspired visual ego-rotation sensor for MAVs," Biological cybernetics, vol. 106(1), pp. $51-63,2012$. 
[40] R. Harrison and C. Koch, "A robust analog VLSI motion sensor based on the visual system of the fly," Autonomous Robots, vol. 7(3), pp. 211224, 1999.

[41] S. Liu and A. Usseglio-Viretta, "Fly-like visuomotor responses of a robot using aVLSI motion-sensitive chips," Biological Cybernetics, vol. 85(6), pp. 449-457, 2001.

[42] R. Brinkworth, P. Shoemaker, and D. O'Carroll, "Characterization of a neuromorphic motion detection chip based on insect visual system," in 5th International Conference on Intelligent Sensors, Sensor Networks and Information Processing (ISSNIP), Melbourne, Australia, December 2009, pp. 289-294.

[43] P. Xu, J. Humbert, and P. Abshire, "Analog VLSI implementation of wide-field integration methods," Journal of Intelligent \& Robotic Systems, vol. 64(3), pp. 465-487, 2011.

[44] J. Krammer and C. Koch, "Pulse-based analog VLSI velocity sensors," IEEE Transactions on Circuits and Systems II: Analog and Digital Signal Processing, vol. 44(2), pp. 86-101, 1997.

[45] G. Barrows and C. Neely, "Mixed-mode VLSI optic flow sensors for inflight control of a Micro Air Vehicle," in SPIE : Critical technologies for the future of computing, vol. 4109, 2000, pp. 52-63.

[46] P.-E. Duhamel, N. Perez-Arancibia, G. Barrows, and R. Wood, "Altitude feedback control of a flapping-wing microrobot using an on-board biologically inspired optical flow sensor," in IEEE International Conference on Robotics and Automation (ICRA), Minnesota, USA, May 2012, pp. 4228-4235.

[47] R. Chan, A. Mulla, and K. Stol, "Characterisation of low-cost optical flow sensors," in Proceedings of the IEEE of the Australasian Conference on Robotics and Automation (ACRA), Brisbane, Australia, December 2010, pp. 1-8.

[48] J. D. Jackson, D. W. Callahan, and J. Marstrander, "A rationale for the use of optical mice chips for economic and accurate vehicle tracking," in Proceedings IEEE International Conference on Automation Science and Engineering CASE, Scottsdale, USA, September 2007, pp. 939-944.

[49] H. Dahmen, A. Millers, and H. A. Mallot, "Insect inspired odometry by optic flow recorded with optical mouse chips," in Flying insects and robots, D. Floreano, J. C. Zufferey, M. V. Srinivasan, and C. Ellington, Eds. Berlin: Sringer, 2009, pp. 115-126.

[50] S. Griffiths, J. Saunders, A. Curtis, B. Barber, T. McLain, and R. Beard "Maximizing miniature aerial vehicles," IEEE Robotics \& Automation Magazine, vol. 13, pp. 34-43, 2006.

[51] A. Beyeler, J. C. Zufferey, and D. Floreano, "Vision-based control of near-obstacle flight," Autonomous Robots, vol. 27(3), pp. 201-219, 2009.

[52] F. Expert, S. Viollet, and F. Ruffier, "A mouse sensor and a 2-pixel motion sensor exposed to continuous illuminance changes," in IEEE Sensors 2011 Conference, Limerick, Ireland, October 2011, pp. 974 977.

[53] F. Aubépart and N. Franceschini, "Bio-inspired optic flow sensors based on FPGA: Application to Micro-Air Vehicles," Microprocessors and Microsystems, vol. 31, pp. 408-419, 2007.

[54] F. Ruffier, S. Viollet, S. Amic, and N. Franceschini, "Bio-inspired optical flow circuits for the visual guidance of micro-air vehicles," in IEEE International Symposium on Circuits and Systems (ISCAS), vol. 3, Bangkok, Thailand, May 2003, pp. 846-849.

[55] M. Pudas, S. Viollet, F. Ruffier, A. Kruusing, S. Amic, S. Leppävuori, and N. Franceschini, "A miniature bio-inspired optic flow sensor based on low temperature co-fired ceramics (ltcc) technology," Sensors and Actuators A: Physical, vol. 133(1), pp. 88-95, 2007.

[56] S. Viollet, F. Ruffier, T. Ray, M. Menouni, F. Aubépart, L. Kerhuel, and N. Franceschini, "Characteristics of three miniature bio-inspired optic flow sensors in natural environments," in Fourth International Conference on Sensor Technologies and Applications (SENSORCOMM), Venice, Italy, July 2010, pp. 51-55.

[57] F. Expert, S. Viollet, and F. Ruffier, "Outdoor field performances of insect-based visual motion sensors," Journal of Field Robotics, vol. 28(4), pp. 974-977, October 2011.

[58] F. L. Roubieu, F. Expert, M. Boyron, B.-J. Fuschlock, S. Viollet, and F. Ruffier, "A novel 1-gram insect based device measuring visual motion along 5 optical directions," in IEEE Sensors Conference, Limerick, Ireland, October 2011, pp. 687-690.

[59] F. Ruffier and F. Expert, "Visual motion sensing onboard a 50-g helicopter flying freely under complex vicon-lighting conditions," in IEEE International Conference on Complex Medical Engineering (CME), Kobe, Japan, July 2012, pp. 634-639.

[60] F. Expert, F. Roubieu, and F. Ruffier, "Interpolation based "time of travel" scheme in a visual motion sensor using a small $2 \mathrm{~d}$ retina," in IEEE Sensors Conference, Taipei, Taiwan, October 2012, pp. 22312234.
[61] K. Götz, "Optomotorische untersuchung des visuellen systems einiger augenmutanten der fruchtfliege drosophila," Biological Cybernetics, vol. 2(2), pp. 77-92, 1964.

[62] N. Franceschini and K. Kirschfeld, "In vivo optical study of photoreceptor elements in the compond eye of drosophila," Biological Cybernetics, vol. 8(1), pp. 1-13, 1971.

[63] D. G. Stavanga, "Angular and spectral sensitivity of fly photoreceptor. I. integrated facet lens and rhabdomere optics," Journal of Comparative Physiology A: Neuroethology, Sensory, Neural, and Behavioral Physiology, vol. 189(1), pp. 1-17, 2003.

[64] J.-M. Pichon, "Guidage visuel d'un robot mobile autonome d'inspiration bionique," Tech. Rep., 1991.

[65] N. Franceschini, F. Ruffier, J. Serres, and S. Viollet, Aerial vehicles. Vienna: In-Tech, 2009, ch. 35 : Optic flow based visual guidance: from flying insects to miniature aerial vehicles, pp. 747-770.

[66] M. F. Land, "Visual acuity in insects," Annual Review of Entomology, vol. 42(1), pp. 147-177, 1997.

[67] G. Sabiron, P. Chavent, T. Raharijaona, P. Fabiani, and F. Ruffier, "Low-speed optic-flow sensor onboard an unmanned helicopter flying outside over fields," in IEEE International Conference on Robotics and Automation (ICRA), 2013 (submitted).

[68] F. Valette, F. Ruffier, S. Viollet, and T. Seidl, "Biomimetic optic flow sensing applied to a lunar landing scenario," in Proceedings of IEEE International Conference on Robotics and Automation (ICRA), Anchorage, USA, May 2010, pp. 2253-2260. 